\title{
Fallopian Tube Cancer with Palmar Fibromatosis or Fasciitis without Polyarthritis
}

\author{
Shoichi Fukui ${ }^{1}$, Sho Fujiwara ${ }^{1}$, Satomi Kobayashi ${ }^{1}$, Satoshi Kamei ${ }^{1}$, Yasunobu Takizawa ${ }^{1}$, \\ Keigo Setoguchi ${ }^{1}$, Masaya Uno ${ }^{2}$ and Nobuaki Funata ${ }^{3}$
}

\begin{abstract}
A 64-year-old Japanese woman had rapidly progressing bilateral palmar contracture associated with severe pain on both palms over the previous 8 weeks, without a history of arthritis in any joints. We suspected palmar fibromatosis or fasciitis without polyarthritis. Because palmar fibromatosis is known to be associated with cancer, we performed cancer screening and the patient was subsequently diagnosed with fallopian tube cancer. This is the first case report of palmar fibromatosis or fasciitis without polyarthritis associated with fallopian tube cancer. The characteristic rapid progression of palmar contracture is a key finding that suggests the potential existence of a malignancy.
\end{abstract}

Key words: fallopian tube cancer, palmar fasciitis and polyarthritis syndrome, palmar fibromatosis, palmar nodules, swollen hand

(Intern Med 54: 2409-2414, 2015)

(DOI: 10.2169/internalmedicine.54.4551)

\section{Introduction}

Various musculoskeletal symptoms may be triggered by the occurrence of a malignancy. These conditions are known as paraneoplastic syndromes. Physicians frequently encounter patients with arthralgia, musculoskeletal pain, and/or skin eruptions, and the symptoms that are associated with cancer must be distinguished from those of rheumatic diseases.

Palmar fibromatosis or fasciitis is sometimes associated with malignancy (1). This disease has a characteristic clinical course that involves rapidly progressing contracture of the palm with associated severe pain. This is a rare disease, however, it is important for physicians to be able to recognize the disease in the patients with these characteristic clinical symptoms and to screen for malignancy. This report presents a clinical case of palmar fibromatosis which led to the diagnosis of fallopian tube cancer.

\section{Case Report}

A 64-year-old Japanese woman was admitted to our hospital due to persistent stiffness in both hands over the previous 8 weeks. Her bilateral palms partially thickened and nodules gradually appeared. She felt severe pain in the palm and it became difficult to flex and extend her fingers. Her primary care doctor prescribed a daily dose of $10 \mathrm{mg}$ of prednisolone, but her symptoms progressively worsened.

The patient's medical history included iron deficiency anemia and a habit of smoking 20 cigarettes a day for 6 years from the age of 30 to 35 years. Her family history included esophageal cancer on her father's side and colon cancer on her mother's side.

On the physical examination at our institution, the patient had tight and disabling flexion contracture of her fingers with marked tethering of the flexor tendons, as is seen in Dupuytren's disease (Fig. 1). She had redness and edema of the skin on her fingers, and elastic hard nodules were present along the flexor tendons. She felt severe tenderness in

\footnotetext{
${ }^{1}$ Department of Rheumatology, Tokyo Metropolitan Cancer and Infectious Diseases Center Komagome Hospital, Japan, ${ }^{2}$ Department of Gynecology, Tokyo Metropolitan Cancer and Infectious Diseases Center Komagome Hospital, Japan and ${ }^{3}$ Department of Pathology, Tokyo Metropolitan Cancer and Infectious Diseases Center Komagome Hospital, Japan

Received for publication November 20, 2014; Accepted for publication January 28, 2015

Correspondence to Dr. Shoichi Fukui, fukui-ngs@umin.ac.jp
} 

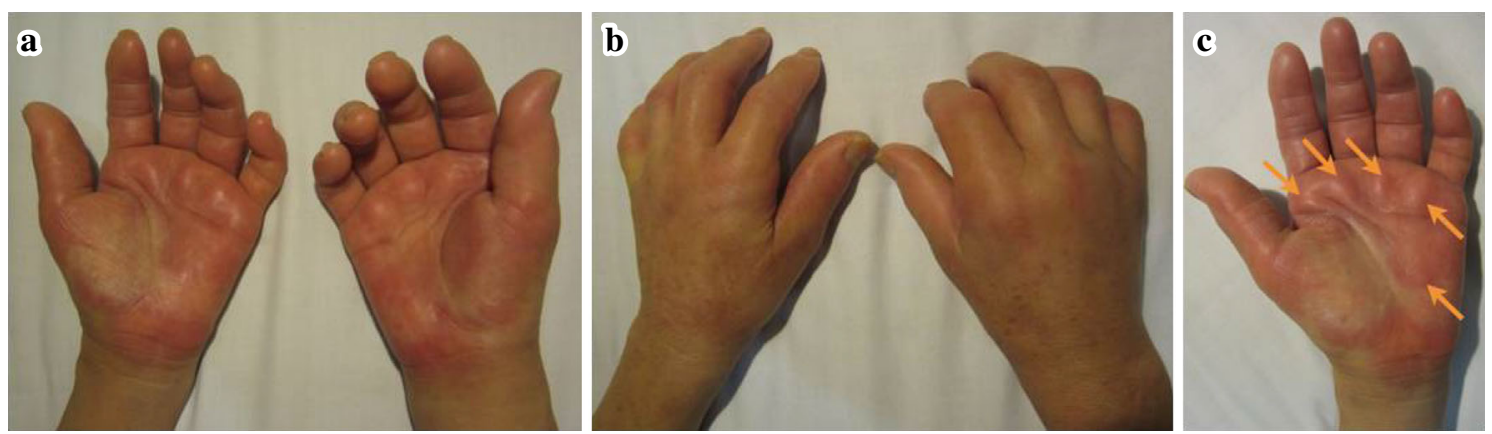

Figure 1. a: The tight and disabling flexion contracture of the patient's fingers with marked tethering of the flexor tendons, as seen in Dupuytren's disease. b: The finger extension was also impaired. c: There was redness on the skin of the patient's fingers and edema surrounding the elastic hard nodules (arrows) along the flexor tendons.

Table. Laboratory Test Results.

\begin{tabular}{clrlrlrl}
\hline & Norm & Result & Unit & & Norm & Result & Unit \\
\hline WBC & $3,500-9,100$ & 8,100 & $/ \mu \mathrm{L}$ & LDH & $119-229$ & 174 & $\mathrm{IU} / \mathrm{L}$ \\
Seg & $40-60$ & 75 & $\%$ & ALP & $115-359$ & 111 & $\mathrm{IU} / \mathrm{L}$ \\
Ly & $25-50$ & 15 & $\%$ & CRP & $<0.30$ & 0.18 & $\mathrm{mg} / \mathrm{dL}$ \\
Mo & $1-14$ & 9 & $\%$ & IgG & $870-1,700$ & 1205 & $\mathrm{mg} / \mathrm{dL}$ \\
Eo & $0-5$ & 1 & $\%$ & IgA & $110-410$ & 145 & $\mathrm{mg} / \mathrm{dL}$ \\
RBC & $3.76-5.00$ & 4.6 & $/ \mu \mathrm{L}$ & IgM & $35-220$ & 140 & $\mathrm{mg} / \mathrm{dL}$ \\
Hb & $11.3-15.2$ & 14.9 & $\mathrm{~g} / \mathrm{dL}$ & $\mathrm{C} 3$ & $65-135$ & 49.8 & $\mathrm{mg} / \mathrm{dL}$ \\
MCV & $79.0-100.0$ & 89.1 & $\mathrm{fl}$ & $\mathrm{C} 4$ & $13-35$ & 16.2 & $\mathrm{mg} / \mathrm{dL}$ \\
MCH & $26.3-34.3$ & 32.4 & $\mathrm{pg}$ & CH50 & $30-45$ & 66.4 & $\mathrm{CH} 50 / \mathrm{mL}$ \\
MCHC & $30.7-36.6$ & 36.3 & $\mathrm{~g} / \mathrm{dL}$ & HbAlc & $4.3-5.8$ & 4.9 & $\%$ \\
PLT & $13.0-36.9$ & 27.4 & $/ \mu \mathrm{L}$ & ANA & $<\times 40$ & $<\times 40$ & \\
TP & $6.7-8.3$ & 7.5 & $\mathrm{~g} / \mathrm{dL}$ & RF & $<15$ & $<15$ & $\mathrm{IU} / \mathrm{mL}$ \\
ALB & $4.0-5.0$ & 4.2 & $\mathrm{~g} / \mathrm{dL}$ & ACPA & $<4.5$ & $<4.5$ & $\mathrm{IU} / \mathrm{mL}$ \\
UN & $8-22$ & 21 & $\mathrm{mg} / \mathrm{dL}$ & MMP-3 & $17.3-59.7$ & 75 & $\mathrm{ng} / \mathrm{mL}$ \\
Cr & $0.4-1.1$ & 0.6 & $\mathrm{mg} / \mathrm{dL}$ & & & & \\
AST & $13-33$ & 13 & $\mathrm{IU} / \mathrm{L}$ & & & & \\
ALT & $8-42$ & 14 & $\mathrm{IU} / \mathrm{L}$ & & & & \\
\hline
\end{tabular}

WBC: white blood cell count, Seg: neutrophilic granulocyte segmented form, Ly: lymphocytes, Mo: monocytes, Eo: eosinophils, RBC: red blood cell count, Hb: hemoglobin, MCV: mean corpuscular volume, $\mathrm{MCH}$ : mean corpuscular hemoglobin, $\mathrm{MCHC}$ : mean corpuscular hemoglobin concentration, PLT: platelet count, UN: nitrogen, Cr: creatinine, AST: aspartate aminotransferase, ALT: alanine aminotransferase, LDH: lactate dehydrogenase, ALP: alkaline phosphatase, CRP: C-reactive protein, HbAlc: hemoglobin Alc, ANA: antinuclear antibodies, RF: rheumatoid factor, ACPA: anti-citrullinated peptide antibody, MMP-3: matrix metalloproteinase-3

her bilateral palm, however, there was no tenderness in the finger joints. Furthermore, the swelling of the finger joints was not prominent. She was unable to flex or extend her fingers, and this symptom had rapidly progressed during the previous 8 weeks.

The patient's body temperature at the initial examination was $37.1^{\circ} \mathrm{C}$. Chest auscultation revealed no adventitious sounds, and her abdomen was slightly swollen. There was no evidence of edema in her lower legs, skin eruptions, swollen joints, skin thickening, Raynaud's phenomenon or abnormal nailfold capillaries.

Table shows the patient's laboratory test results including the following: C-reactive protein level, $0.18 \mathrm{mg} / \mathrm{dL}$; rheumatoid factor, <15 IU/mL; anti-cyclic citrullinated peptide (CCP) antibody, <4.5 IU/mL; anti-nuclear antibody, 40 titer; hemoglobin A1c, 4.9\%; and matrix metalloproteinase-3, 75 ng/mL. Disease-specific antibody testing (e.g., anti-Scl-70 antibody and anti-centromere antibody) was negative. A urinalysis revealed neither hematuria nor proteinuria.

Superficial ultrasonography showed hypoechoic nodules above the flexor tendons. Power Doppler signals of the nodules were positive (Fig. 2), however, there was no evidence of arthritis because there was no increased synovia in the finger joints.

Magnetic resonance imaging (MRI) with T1 and T2 imaging showed high-intensity nodules above the flexor tendons. Short T1 inversion recovery (STIR) imaging also showed high-intensity nodules above the flexor tendons and high-intensity fascia (Fig. 3). However, there was no evidence on MRI suggestive of the existence of arthritis.

Due to the unusual clinical findings and the rapidly progressive palmar contracture, we suspected palmar fibromatosis or fasciitis, which is sometimes associated with malignancy. We thus screened the patient for malignant diseases. 


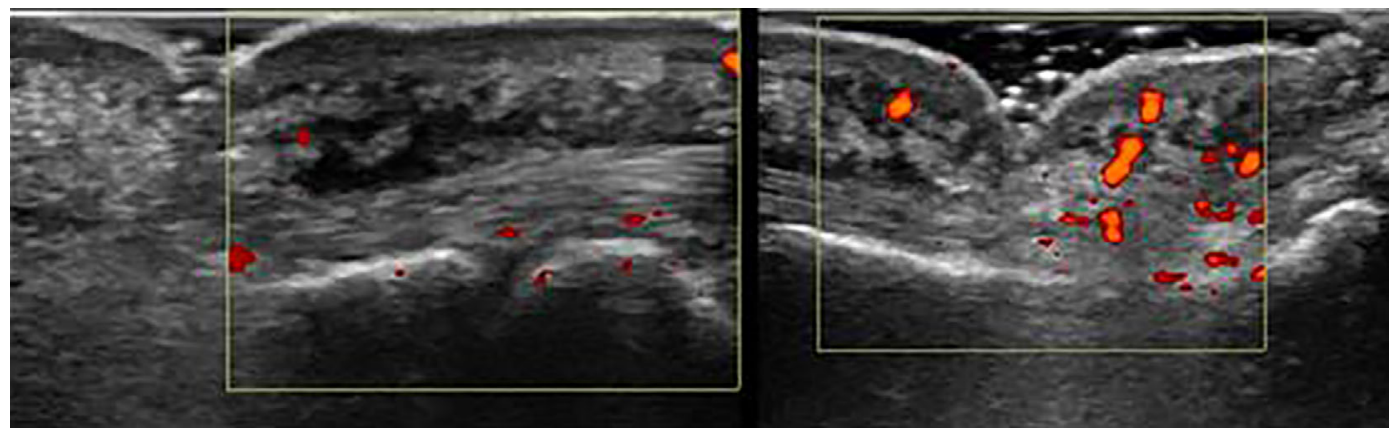

Figure 2. Superficial ultrasonography revealed hypoechoic nodules above the flexor tendons. The power Doppler signal was positive.
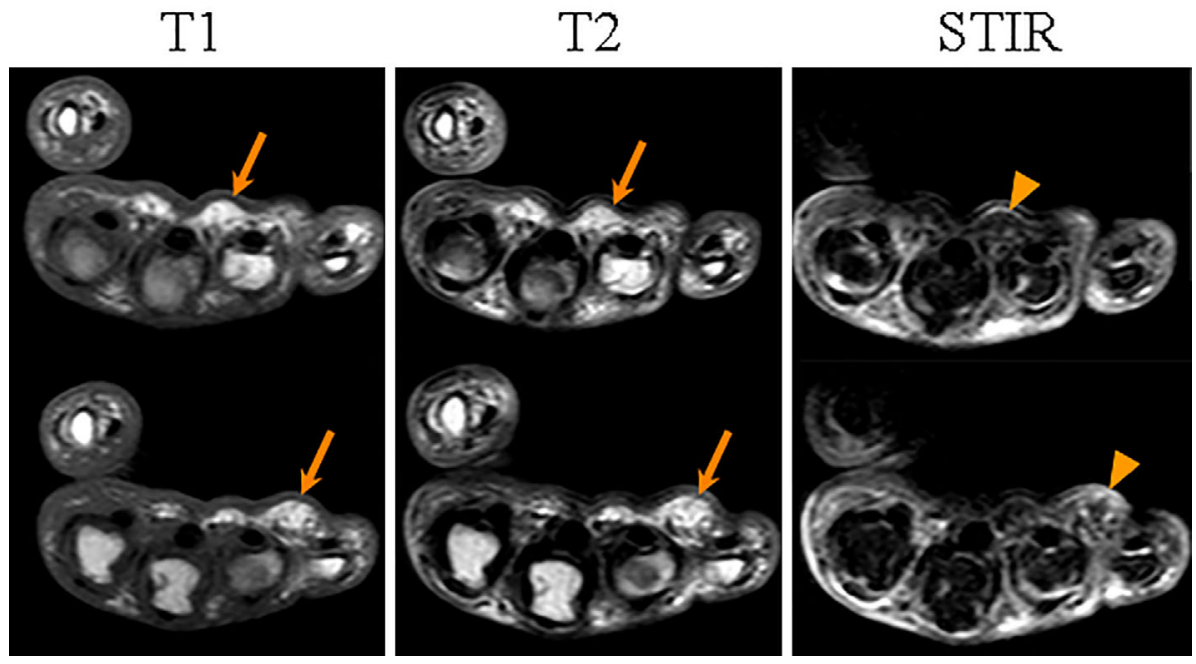

Figure 3. MRI with T1, T2, and STIR imaging showing high-intensity nodules above the flexor tendons (arrows) and high-intensity fascia (arrowheads).

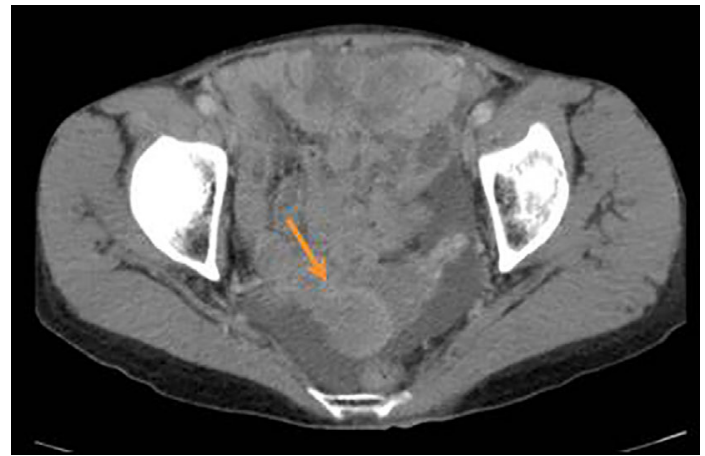

Figure 4. Pelvic CT showing a swollen right ovary (see arrow) and a small amount of ascites.

Her titer of cancer antigen 125 (CA 125) was 688 U/L (normal range $<35 \mathrm{U} / \mathrm{L}$ ), and pelvic computed tomography (CT) showed a swollen right ovary $(30 \times 35 \mathrm{~mm})$ and a small amount of ascites (Fig. 4).

Because ovarian cancer was suspected, laparotomy was performed. There was a solitary, white and yellowish mixed mass $(55 \times 40 \times 25 \mathrm{~mm})$ in the right fallopian tube which had invaded the right ovary and uterus (Fig. 5). A metastatic tumor $(15 \mathrm{~mm}$ dia.) was located in the greater omentum.

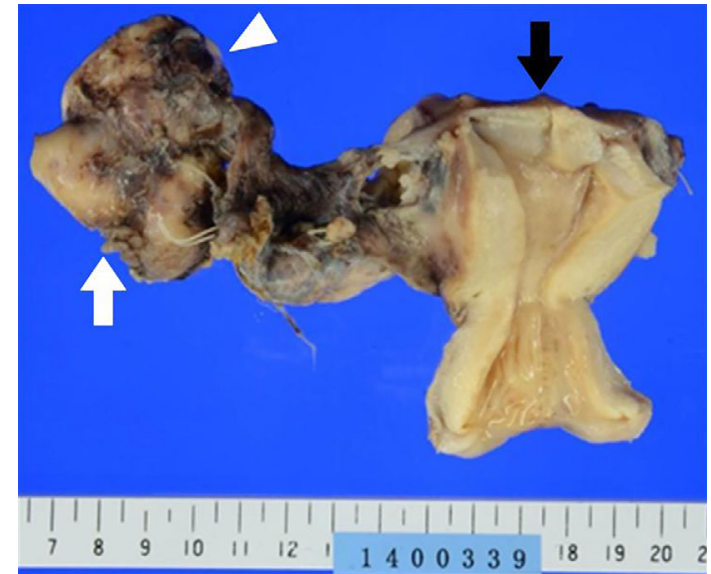

Figure 5. A resected specimen with a solitary white and yellowish mixed mass on the right fallopian tube (white arrowhead) that invaded the right ovary (white arrow) and the uterus (black arrow) is shown.

Serous, poorly differentiated cells with atypical nuclei pathologically proliferated in the right fallopian tube (Fig. 6). They were associated with small lumens and slit-like spaces (Fig. 7). Bone scintigraphy and CT imaging did not show 


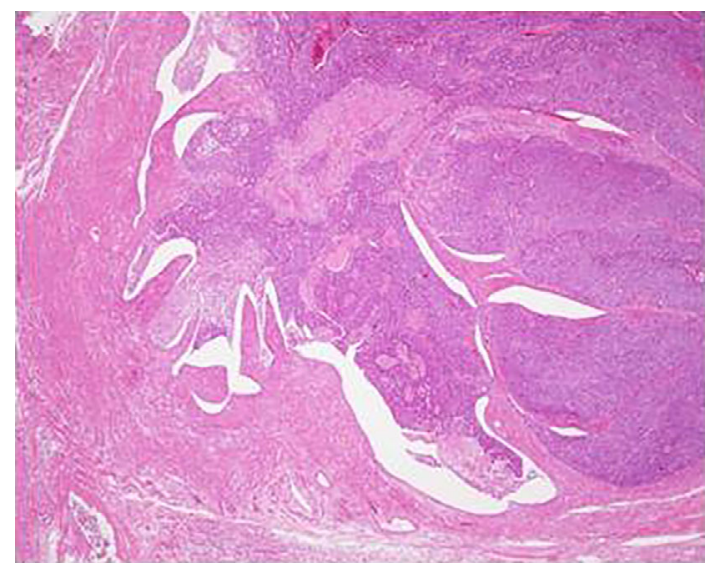

Figure 6. A section of the fallopian tube was obtained for a histological examination (Hematoxylin and Eosin staining, 40x). Serous, poorly differentiated cells with atypical nuclei proliferated.

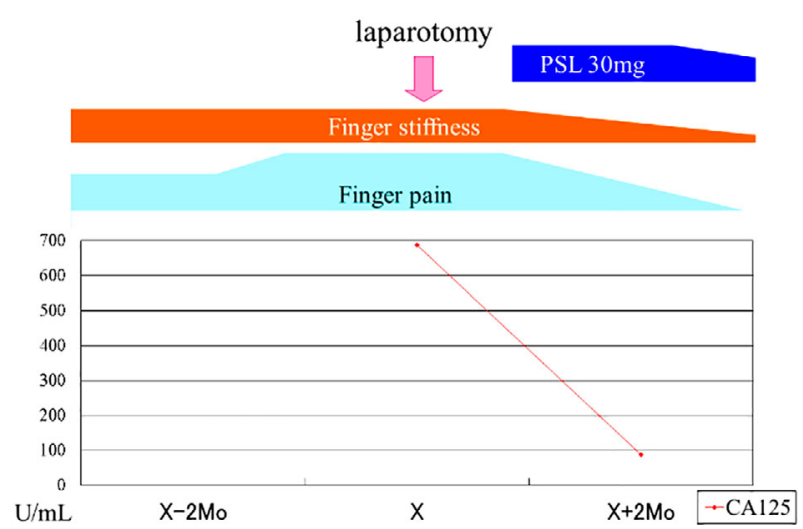

Figure 8. The clinical course of the patient.

any evidence of bone metastases or other metastases. We therefore diagnosed the serous fallopian tube cancer at pT3cNxM0 stage IIIC with palmar fibromatosis.

The patient was treated with chemotherapy using carboplatin and paclitaxel; however, her symptoms did not improve. After the removal of the fallopian tube cancer and the initiation of chemotherapy, we started the patient on a 30$\mathrm{mg}$ daily prednisolone regimen and rehabilitation for her fingers. The tenderness in her palms then disappeared and the stiffness and contracture of her palms were mildly relieved (Fig. 8).

\section{Discussion}

We herein present a case of palmar fibromatosis or fasciitis without polyarthritis associated with fallopian tube cancer. It is often difficult to distinguish fibromatosis and fasciitis without biopsy findings because both diseases present with thickening of the palmar fascia and flexion contracture. Fibromatosis is characterized by fibroblastic proliferations with spindle-shaped myofibroblastic cells, dense deposits of intercellular collagen fibers, variable amounts of extracellu-

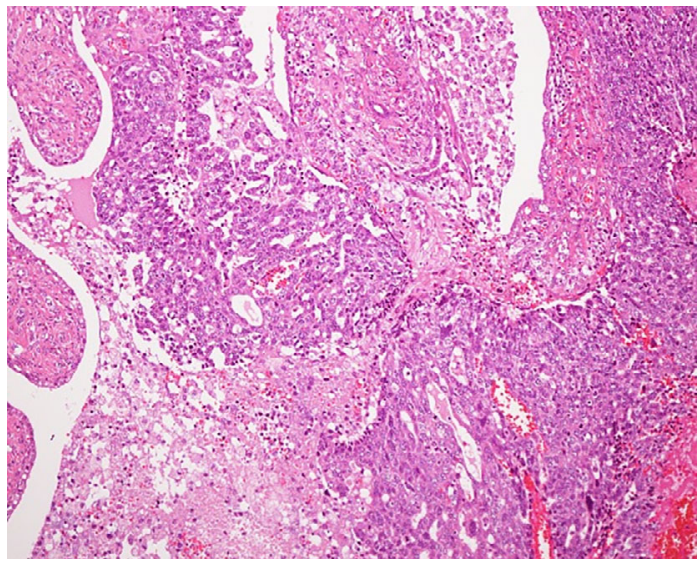

Figure 7. A section of the fallopian tube was obtained for a histological examination (Hematoxylin and Eosin staining, 400x). There were small lumens and slit-like spaces.

lar myxoid matrix, and compressed and elongated vessels (2). Palmar fibromatosis, also referred to as Dupuytren's disease, is the most common of the superficial fibromatoses. To the best of our knowledge, only one previous case of ovarian cancer with palmar fibromatosis has been reported (3).

Palmar fasciitis is known to be associated with cancer. In 1967, Bremer reported a patient with palmar fasciitis and arthritis of the finger joints associated with metastatic ovarian cancer (4). In 1982, Medsger et al. reported six cases of palmar fasciitis and polyarthritis associated with ovarian cancer (5). This series of conditions came to be referred to as cases of palmar fasciitis and polyarthritis syndrome (PFPAS). There are some case reports of PFPAS associated with breast cancer (6), lung cancer (7), or gastric cancer (8). Only two cases of PFPAS with fallopian tube cancer have been previously reported $(9,10)$. Additionally, in some case reports, the terms "fibromatosis" and "fasciitis" are used interchangeably (11-13).

The MRI findings of fibromatosis or fasciitis vary with T1- and T2-weighted signal intensity, which depends on the lesion cellularity. The lesions with higher collagen levels demonstrate low-intensity signals, and the lesions with higher cellularity demonstrate high-intensity signals (14). Because T1, T2, and STIR imaging revealed high-intensity lesions in the present case, we expected the lesions to have a higher cellularity. Superficial ultrasonography showed hypoechoic lesions with a positive power Doppler signal. This observation is compatible with palmar fibromatosis (14). Although we speculate that our patient had palmar fibromatosis, it is difficult to distinguish palmar fibromatosis and palmar fasciitis strictly in the absence of pathological evidence. In addition, a biopsy of the palm or tendon is difficult because it can affect the functional ability of the fingers or disturb sensation. We were thus unable to make a conclusion regarding whether palmar fibromatosis or fasciitis was the diagnosis.

In our patient, there was no evidence of clinical arthritis 
because there was no increased synovia of the finger joints on both superficial ultrasonography and MRI. This is a very important finding. We conducted a PubMed (http://www. ncbi.nlm.nih.gov/pubmed) database search to find cases of palmar fibromatosis or fasciitis without polyarthritis associated with cancer. Our search of reports from 1944 to 2014 with the search terms "palmar fibromatosis," "palmar fasciitis," "cancer," "neoplasm," "carcinoma," "malignancy," and "Dupuytren's disease" returned only four cases of palmar fibromatosis or fasciitis without polyarthritis associated with cancer [two ovarian cancers $(3,15)$, one breast cancer (16), and one non-small cell lung cancer (17)]. Therefore, we cannot determine whether palmar fibromatosis or fasciitis without polyarthritis is a paraneoplastic syndrome or not based solely on these four cases. However, even if the patients with palmar fibromatosis or fasciitis do not have polyarthritis, the potential existence of malignancy should not be ignored. The characteristic rapid progression of the palmar contracture is a key finding. It was previously noted that palmar fasciitis associated with cancer progresses much more rapidly than in Dupuytren's disease (16).

In some cases, the resection of the underlying cancer may stop the progression of the symptoms and relieve the symptoms $(8,18,19)$. Only rarely do glucocorticoids produce a favorable effect $(18,20-22)$. In the present patient, the symptoms of pain, stiffness and contracture were alleviated by glucocorticoid therapy.

The pathogenesis of palmar fibromatosis and fasciitis is not clearly understood. The role of soluble stimulators of fibroblast activity such as transforming growth factor $\beta$ (TGF$\beta$ ) or connective tissue growth factor has been reported $(5,23,24)$. However, because the relationship between Dupuytren's disease and TGF- $\beta$ had also been shown (25), differences in the TGF- $\beta$ levels cannot explain the different etiologies of palmar fibromatosis or fasciitis associated with cancer and Dupuytren's disease. A higher level of TGF- $\beta$ may be released from cancer and facilitate the rapid progression in the patients with palmar fibromatosis or fasciitis associated with cancer.

Manger et al. reviewed the cases of 100 patients with PFPAS and found that of the 87 patients with PFPAS associated with cancer, 32 patients had ovarian cancer and two patients had fallopian tube cancer (1). There are many similarities between fallopian tube cancer and ovarian cancer, and ovarian cancer is believed to originate in the distal fallopian tube (26). Ovarian cancer releases many types of cytokines such as TGF- $\beta$, insulin-like growth factor, fibroblast growth factor, epidermal growth factor and vascular endothelial growth factor (27). In the present patient, these cytokines may have played an important role in the emergence of palmar fibromatosis or fasciitis.

An explanation for the absence of polyarthritis in the present patient may be due to different cytokine profiles. In rheumatoid arthritis, it is known that tumor necrosis factoralpha (TNF- $\alpha$ ) and interleukin-6 (IL-6) are associated with active synovitis (28). It has also been reported that some ovarian cancers release TNF- $\alpha$ (29), and a variety of ovarian cancer cell lines showed different IL-6 responses to TNF- $\alpha$ stimulation (30). In the present patient, the fallopian tube cancer may have had low levels of TNF- $\alpha$ and IL-6, which accounted for the absence of polyarthritis. However, we could not test this hypothesis by obtaining evidence, such as the patient's serum concentration of cytokines or immunostaining of the biopsy specimens. Further investigation into the mechanisms of palmar fibromatosis is therefore required.

In the present patient, we rapidly diagnosed and treated the fallopian tube cancer in light of the characteristic rapid progression of palmar contracture. It is important to differentiate paraneoplastic syndromes when familiar rheumatic diseases may not adequately account for a patient's clinical symptoms. The commitment of the attending physicians to become familiar with and identify rare diseases can therefore improve the prognoses of their patients.

\section{The authors state that they have no Conflict of Interest (COI).}

\section{References}

1. Manger B, Schett G. Palmar fasciitis and polyarthritis syndrome: systematic literature review of 100 cases. Semin Arthritis Rheum 44: 105-111, 2014.

2. Weiss SW, Goldblum JR, Enzinger FM. Fibromatoses. In: Enzinger and Weiss's Soft Tissue Tumors. 5th ed. Weiss SW, Goldblum JR, Eds. Mosby Elsevier, Philadelphia, 2008: 227-228.

3. Alexandroff AB, Hazleman BL, Matthewson M, et al. Woody hands. Lancet 361: 1344, 2003.

4. Bremer C. Shoulder-hand syndrome. A case of unusual aetiology. Ann Phys Med 9: 168-171, 1967.

5. Medsger TA, Dixon JA, Garwood VF. Palmar fasciitis and polyarthritis associated with ovarian carcinoma. Ann Intern Med 96: 424-431, 1982.

6. Valverde-Garcia J, Juanola-Roura X, Ruiz-Martin JM, Nolla-Sole JM, Rodriguez-Moreno J, Roig-Escofet D. Paraneoplastic palmar fasciitis-polyarthritis syndrome associated with breast cancer. J Rheumatol 14: 1207-1209, 1987.

7. Sheehy C, Ryan JG, Kelly M, Barry M. Palmar fasciitis and polyarthritis syndrome associated with non-small-cell lung carcinoma. Clin Rheumatol 26: 1951-1953, 2007.

8. Enomoto M, Takemura H, Suzuki M, et al. Palmar fasciitis and polyarthritis associated with gastric carcinoma: complete resolution after total gastrectomy. Intern Med 39: 754-757, 2000.

9. Taggart AJ, Iveson JM, Wright V. Shoulder-hand syndrome and symmetrical arthralgia in patients with tubo-ovarian carcinoma. Ann Rheum Dis 43: 391-393, 1984.

10. Denschlag D, Riener E, Vaith P, Tempfer C, Keck C. Palmar fasciitis and polyarthritis as a paraneoplastic syndrome associated with tubal carcinoma: a case report. Ann Rheum Dis 63: 11771178, 2004.

11. Champion GD, Saxon JA, Kossard S. The syndrome of palmar fibromatosis (fasciitis) and polyarthritis. J Rheumatol 14: 11961198, 1987.

12. Cox NH, Ramsay B, Dobson C, Comaish JS. Woody hands in a patient with pancreatic carcinoma: a variant of cancer-associated fasciitis-panniculitis syndrome. Br J Dermatol 135: 995-998, 1996.

13. Pfinsgraff J, Buckingham RB, Killian PJ, et al. Palmar fasciitis and arthritis with malignant neoplasms: a paraneoplastic syndrome. Semin Arthritis Rheum 16: 118-125, 1986. 
14. Walker EA, Petscavage JM, Brian PL, Logie CI, Montini KM, Murphey MD. Imaging features of superficial and deep fibromatoses in the adult population. Sarcoma 2012: 215810, 2012.

15. Strobel ES, Lacour M, Peter HH. Palmar fascial thickening and contractures of fingers resembling arthritis: a paraneoplastic symptom? Rheumatol Int 12: 79-80, 1992.

16. Sandhya P, Danda D. Paraneoplastic palmar fasciitis in carcinoma breast. J Clin Rheumatol 18: 112, 2012.

17. McGivern U, McAleese J. Palmar fasciitis: a para-neoplastic phenomenon indicating recurrence of non small cell lung cancer: case report and review of the literature. Int $J$ Rheum Dis 15: e8-e9, 2012.

18. Goldberg E, Dobransky R, Gill R. Reflex sympathetic dystrophy associated with malignancy. Arthritis Rheum 28: 1079-1080, 1985.

19. Sen J, Clewes AR, Quah SA, Hiscott PS, Bucknall RC, Damato BE. Presymptomatic diagnosis of bronchogenic carcinoma associated with bilateral diffuse uveal melanocytic proliferation. Clin Experiment Ophthalmol 34: 156-158, 2006.

20. Baron M. Palmar fasciitis, polyarthritis, and carcinoma. Ann Intern Med 97: 616, 1982.

21. Roman S, Tebib J, Scoazec JY, Menard Y, Paliard P, Dumortier J. [Palmar fasciitis and paraneoplastic polyarthritis associated with hepatocellular carcinoma]. Gastroenterol Clin Biol 25: 203-204, 2001 (in French, Abstract in English).

22. Virik K, Lynch KP, Harper P. Gastroesophageal cancer, palmar fasciitis and a matrix metalloproteinase inhibitor. Intern Med J 32: 50-51, 2002.

23. Clarke LL, Kennedy CT, Hollingworth P. Palmar fasciitis and polyarthritis syndrome associated with transitional cell carcinoma of the bladder. J Am Acad Dermatol 64: 1159-1163, 2011.

24. Mahoney K, Cayea D, Li QK, Gelber AC. A handy clue: palmar fasciitis and polyarthritis syndrome. Am J Med 127: 116-118, 2014.

25. Kloen P. New insights in the development of Dupuytren's contracture: a review. Br J Plast Surg 52: 629-635, 1999.

26. Lee Y, Miron A, Drapkin R, et al. A candidate precursor to serous carcinoma that originates in the distal fallopian tube. J Pathol 211: 26-35, 2007.

27. Scheinfeld N. A review of the cutaneous paraneoplastic associations and metastatic presentations of ovarian carcinoma. Clin Exp Dermatol 33: 10-15, 2008.

28. Alghasham A, Rasheed $Z$. Therapeutic targets for rheumatoid arthritis: progress and promises. Autoimmunity 47: 77-94, 2014.

29. Abbate I, Correale M, Gargano G, et al. Tumor necrosis factor and soluble interleukin-2 receptor: two immunological biomarkers in female neoplasms. Eur J Gynaecol Oncol 13 (1 Suppl): 92-96, 1992.

30. Asschert JG, Vellenga E, Ruiters MH, de Vries EG. Regulation of spontaneous and TNF/IFN-induced IL-6 expression in two human ovarian-carcinoma cell lines. Int J Cancer 82: 244-249, 1999.

(C) 2015 The Japanese Society of Internal Medicine http://www.naika.or.jp/imonline/index.html 